\title{
Spectropolarimetric analysis of an active region filament.
}

\section{Evidence of the limitations of a single-component model}

\author{
C. J. Díaz Baso ${ }^{1,2,3}$, M. J. Martínez González ${ }^{1,2}$, and A. Asensio Ramos ${ }^{1,2}$ \\ 1 Instituto de Astrofísica de Canarias, C/Vía Láctea s/n, 38205 La Laguna, Tenerife, Spain \\ 2 Departamento de Astrofísica, Universidad de La Laguna, 38206 La Laguna, Tenerife, Spain \\ 3 Institute for Solar Physics, Dept. of Astronomy, Stockholm University, AlbaNova University Centre, 10691 Stockholm, Sweden \\ e-mail: carlos.diaz@astro.su.se
}

Received 6 December 2018 / Accepted 21 April 2019

\begin{abstract}
Aims. Our aim is to demonstrate the limitations of using a single-component model to study the magnetic field of an active region filament. To do this, we analyzed the polarimetric signals of the He I $10830 \AA$ multiplet, which were acquired with the infrared spectrograph GRIS of the GREGOR telescope (Tenerife, Spain).

Methods. After a first analysis of the general properties of the filament using Hazel under the assumption of a single-component model atmosphere, in this second part we focus our attention on the observed Stokes profiles and the signatures that cannot be explained with this model.

Results. We have found an optically thick filament whose blue and red components have the same sign in the linear polarization as an indication of radiative transfer effects. Moreover, the circular polarization signals inside the filament show strong magnetic field gradients. We also show that even a filament with such high absorption still shows signatures of the circular polarization that is generated by the magnetic field below the filament. The reason is that the absorption of the spectral line decays very quickly toward the wings, just where the circular polarization has a larger amplitude. In order to separate the two contributions, we explore the possibility of a two-component model, but the inference becomes impossible to overcome because very many solutions are compatible with the observations.
\end{abstract}

Key words. Sun: filaments, prominences - Sun: chromosphere - Sun: magnetic fields - Sun: infrared - Sun: evolution

\section{Introduction}

After some decades of challenging observations of solar filaments and prominences and the development of the theory that explains how polarization signals are generated and modified by the magnetic field (see Bommier et al. 1981, 1989; Landi Degl'Innocenti 1982; Trujillo Bueno et al. 2002; Trujillo Bueno \& Asensio Ramos 2007; Casini et al. 2009a, and references therein), we still lack exact knowledge of the magnetic topology of solar filaments (Labrosse et al. 2010; Mackay et al. 2010). Numerous models and simulations have allowed a better understanding of the formation process and how this plasma is supported against gravity (Kippenhahn \& Schlüter 1957; Kuperus \& Raadu 1974; Antiochos et al. 1999; Xia et al. 2012; Keppens \& Xia 2014), but the most reliable way to obtain quantitative information on its magnetic field and to verify the veracity of the current models is through the analysis of spectropolarimetric observations.

Solar filaments can be observed, among other spectral lines, in the He I $10830 \AA$ multiplet as absorption features. This spectral line has been widely used as a chromospheric diagnostic tool because of its sensitivity to the Hanle and Zeeman effects, and the release of fast and robust inversion codes, such as the Hazel (Asensio Ramos et al. 2008) and HeLIx ${ }^{+}$codes (Lagg et al. 2004, 2009), helped in analyzing this line. This spectral line is commonly optically thin in the solar spectrum, and the opacity is only high enough around specific patches on the solar disk. This multiplet also has several problems. First, the involved polarization signals are weak and are detected only at a level of a few $10^{-3} I_{\mathrm{c}}$ in the better cases. This value corresponds to the typical linear polarization measured in prominences and filaments, but also in other solar regions (Schad et al. 2013). A maximum of $\sim 3.10^{-3} I_{\mathrm{c}}$ is reached even in active region filaments with high horizontal fields (Kuckein et al. 2012). In contrast, Stokes $V$ can reach higher values of about $10^{-2} I_{\mathrm{c}}$ in very highly magnetized regions. Second, magnetic ambiguities lead to different magnetic topologies that generate similar polarimetric signals. These ambiguities can be solved when the configuration is expected to be simple, for instance, in sunspots (Schad et al. 2013; Joshi et al. 2016), where the expected magnetic field is assumed to be nearly radial from the center of the sunspot and aligned with the filaments of the inner penumbra. However, filaments and prominences are very difficult to disambiguate. A good example was shown by Martínez González et al. (2015), who imposed some additional physical constraints on the stability of the structure to be able to reject some of the inferred configurations, or by Orozco Suárez et al. (2014), whose selection was based on the chirality of the filament.

New recent studies have shown the importance of considering more complex models to understand the physical mechanisms behind the formation of the $\mathrm{He}_{\mathrm{I}} 10830 \AA$ multiplet. Observational evidence has been obtained, for example, that the atomic orientation is generated by the Doppler shifted illumination that comes from the underlying magnetized photosphere, 
which explains the existence of extremely asymmetric Stokes $V$ profiles (Martínez González et al. 2012). Other studies have pointed out based on numerical experiments that it is problematic to assume a 1D slab model to infer the magnetic field of complex structures (Milić et al. 2017). The studies emphasized the multidimensional effects that are often neglected in optically thick structures, which were also noted by others based on the ratio between the two components of the helium triplet (López Ariste \& Casini 2002).

Díaz Baso et al. (2016) showed for filaments that are observed on the disk that the polarization signals measured in active region filaments above magnetized regions (Kuckein et al. 2009; Xu et al. 2012) could be subject to biases. The authors pointed out that the observed signals could be explained with a two-component atmospheric model that simulated the filament and the chromospheric region below. This is a very natural way of explaining the observations without relying on other mechanisms such as horizontal illumination in the slab (Trujillo Bueno \& Asensio Ramos 2007) or unresolved magnetic fields (Casini et al. 2009a), which have been proposed to strongly modify the polarization signals.

In the first part of this series (Díaz Baso et al. 2019, hereafter Paper I) we studied high-quality polarimetric observations of an active region filament located above granulation. Even though it was less strongly contaminated by the underlying magnetized areas (a sunspot), a single-component model showed some unrealistic and inconsistent results in the magnetic field vector inference. The Stokes $V$ map of He I did not show any clear signature of the filament, but it was very similar to the circular polarization map of the photosphere. After the one-component inference, the local azimuth map followed the same pattern as was observed in the circular polarization map. This means that Stokes $V$ conditions the inferred magnetic field vector and produces unrealistic results in the spine of the filament (strong shears in the azimuth) that suggest that the Stokes $V$ might come from a fundamentally different place than the linear polarization. In this second part we describe some clear indications that more complex models are required to explain the observed profiles. Simple onecomponent models are not able to fully reproduce the profiles, and more importantly, can lead to biased or unphysical results.

\section{Observations}

The observations were carried out on 17 June 2014 using the GREGOR Infrared Spectrograph (GRIS, Collados et al. 2012) at the GREGOR telescope (Schmidt et al. 2012). We recorded the spectropolarimetric measurements of an active region filament that was located close to the disk center $(\mu=0.92)$. The observed filament was situated close to a sunspot in active region NOAA 12087. The data acquisition and reduction process were described in detail in Paper I. An example of the observed spectra is displayed in Fig. 1. In the upper panel we show the corrected Stokes $I$ spectra obtained as the average profile of a quietSun area. The lower panel shows a slit-spectrum chosen from the third scan at $Y=8^{\prime \prime}$. In the lower part close to $X=12^{\prime \prime}$, we show the effect of the highly dynamic chromosphere on the He I triplet at $10830 \AA$. These types of profiles are found at the border of the filament; a clear second component that is strongly redshifted and has velocities around $30 \mathrm{~km} \mathrm{~s}^{-1}$ is visible as well. These pixels can be easily explained as material falling down from the filament, where the magnetic field is more vertical and cannot keep the material against gravity. This case clearly shows that an additional atmospheric component is required as the components are clearly shifted in wavelength. This scenario has also been
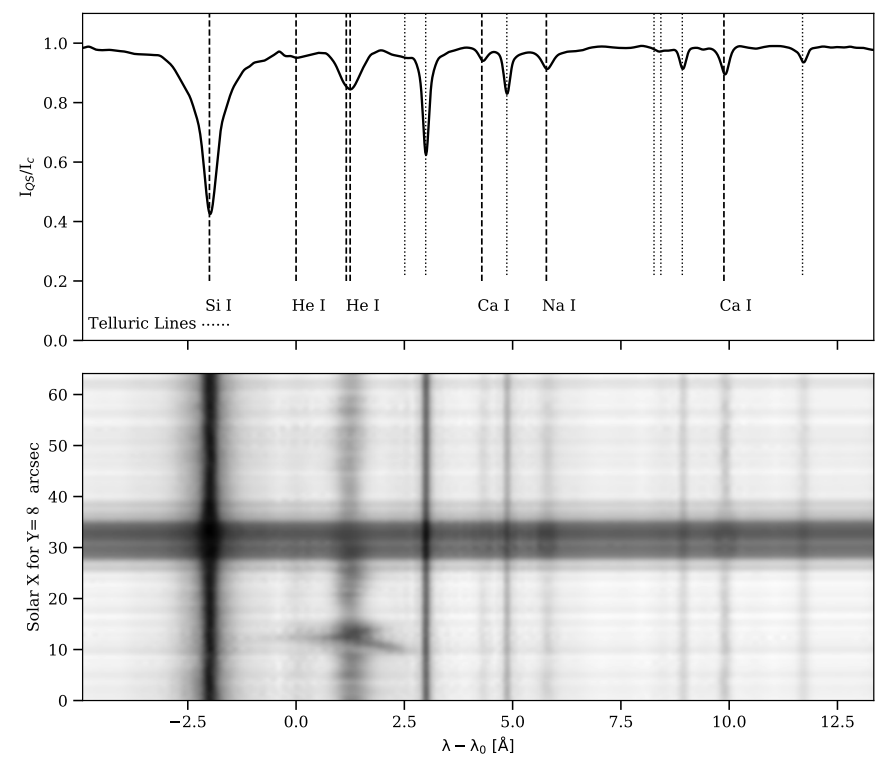

Fig. 1. Upper panel: median intensity spectrum of the quiet Sun normalized to the quiet-Sun continuum. Lower panel: spectra along the slit for the third scanning position. The reference wavelength $\lambda_{0}=$ $10829.0911 \AA$ is the central wavelength of the blue component of the He I $10830 \AA$ multiplet. Close to it, the two red components of the same multiplet are blended in the same profile.

reported by other studies (Sasso et al. 2011; Schad et al. 2016), and it usually occurs in very localized regions of the field of view (FOV). In this study we point out many instances of multicomponent atmospheres in a large portion of the FOV.

In the following sections we therefore analyze in detail the observed profiles and show that a single-component model is not able to reproduce certain aspects in the intensity, linear polarization, and circular polarization profiles. For this purpose we make use of the third scan because the filament has the highest absorption and the features are more pronounced, but similar conclusions can also be drawn from the other scans.

\section{Analysis of Stokes I}

Numerous studies of solar prominences have inferred the physical parameters of the plasma, and in particular, the prominence temperature, by analyzing the shape and intensity of spectral lines when the plasma is optically thin (see review of Parenti 2014, and references therein). However, we demonstrate that our observations show signs of optically thick plasma (López Ariste \& Casini 2002). In the following, we show two consequences of the high absorption in the filament: (i) the differential saturation effect between the red and blue components of the triplet, and (ii) misfits when using the code HAzEL, which can also be explained by radiative transfer effects.

Figure 2 shows the intensity amplitude ratio map between the blue and red components of the He I $10830 \AA$ triplet. Because the lines are in absorption, we modeled each component with a Gaussian function with the same width. When a second absorption profile appeared shifted in wavelength, we fit two sets of Gaussians to remove this new component from the calculations. As the width is roughly the same for the two components of the multiplet, the ratio of amplitudes $\mathcal{R}=A_{\mathrm{B}} / A_{\mathrm{R}}$ is also approximately equal to the ratio of areas. The line ratio between the two resolved $\mathrm{He}$ I components is about 0.4 inside the filament, but it is in the range $0.1-0.2$ in the remaining areas 


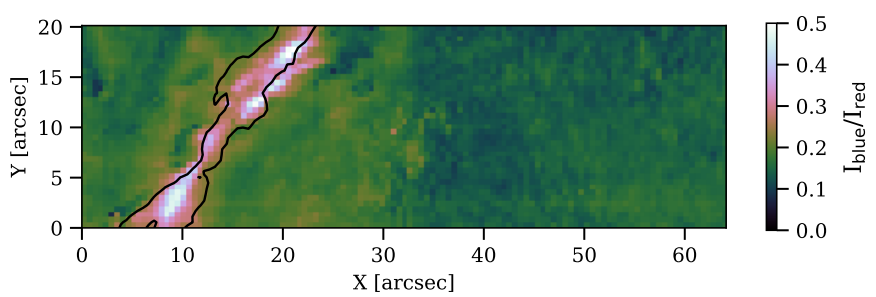

Fig. 2. Ratio between the Stokes $I$ amplitudes of the blue $A_{\mathrm{B}}$ and red $A_{\mathrm{R}}$ component. The contour shows the level $0.4 A_{\mathrm{R}}$, where the red component starts to saturate.
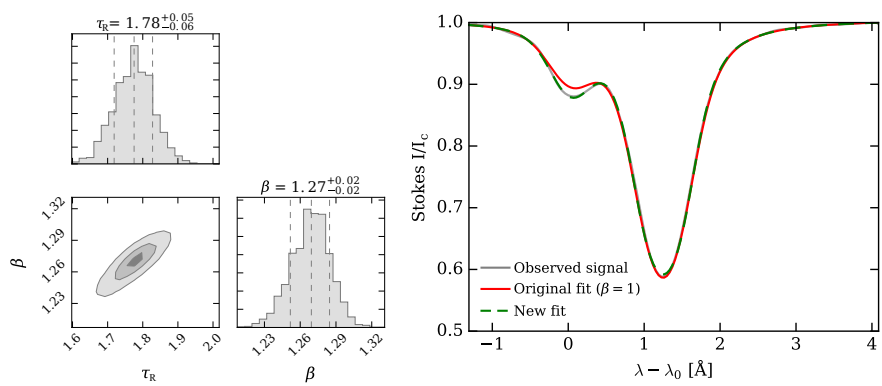

Fig. 3. Bayesian estimation of the $\beta$ parameter. Left panel: posterior probability distribution of $\beta$ and $\tau_{\mathrm{R}}$, and the correlation between them. Right panel: improved fit of the blue component of the $\mathrm{He}_{\mathrm{I}}$ triplet when going from $\tau_{\mathrm{R}}=1.2, \beta=1.0$ (red line) to $\tau_{\mathrm{R}}=1.8, \beta=1.3$ (green line).

(Penn \& Kuhn 1995). This is the value that is expected for an optically thin plasma. This means that we find some indication of saturation only in the filament, with a maximum value of $\mathcal{R}=0.5$.

Another feature that suggests radiative transfer effects is that Hazel systematically produces misfits in the blue component of the multiplet in Stokes $I$. This indicates that the source function used in HAzEL, which comes directly from the lower atmosphere boundary condition, is not appropriate. For this reason, we added a new multiplicative parameter $\beta$ in HAzEL that arbitrarily modifies the source function of the slab $\mathbf{S}$. Consequently, the emergent Stokes profiles are now given by (see Asensio Ramos et al. 2008)

$\mathbf{I}=\mathrm{e}^{-\mathbf{K}^{*} \tau_{\mathrm{R}}} \mathbf{I}_{\text {Sun }}+\left(\mathbf{K}^{*}\right)^{-1}\left(1-\mathrm{e}^{-\mathbf{K}^{*} \tau_{\mathrm{R}}}\right) \beta \mathbf{S}$.

For $\beta<1$ we can mimic a slab whose source function is much smaller than the function determined by the boundary condition. The opposite happens when $\beta>1$, which can also be used to mimic strong emission profiles, like those of flares (Libbrecht et al. 2019). In this equation, $\tau_{\mathrm{R}}$ represents the optical depth measured at the central wavelength of the red blended component of the He I triplet.

All inversions up to now were carried out with $\beta=1$. We explore here the effect of adding $\beta$ in the inversion as an additional free parameter. We show in Fig. 3 an example of such analysis carried out with MultiNest (Feroz et al. 2009) to detect possible ambiguities. It is clear that the addition of $\beta$ results in a much better fit of the blue component of the multiplet (green line in the right panel of Fig. 3). However, $\beta$ and $\tau_{\mathrm{R}}$ are degenerate and have opposite effects in the emergent profile: increasing $\beta$ reduces the absorption, while increasing $\tau_{\mathrm{R}}$ increases the absorption. Therefore, an increase in $\beta$ can be compensated for (up to a point) with an increase in $\tau_{\mathrm{R}}$. In spite of this degeneracy, it is possible to find a pair of $\beta$ and $\tau_{\mathrm{R}}$ that produces a good fit to the Stokes $I$ profile of the multiplet. The general trend is that adding
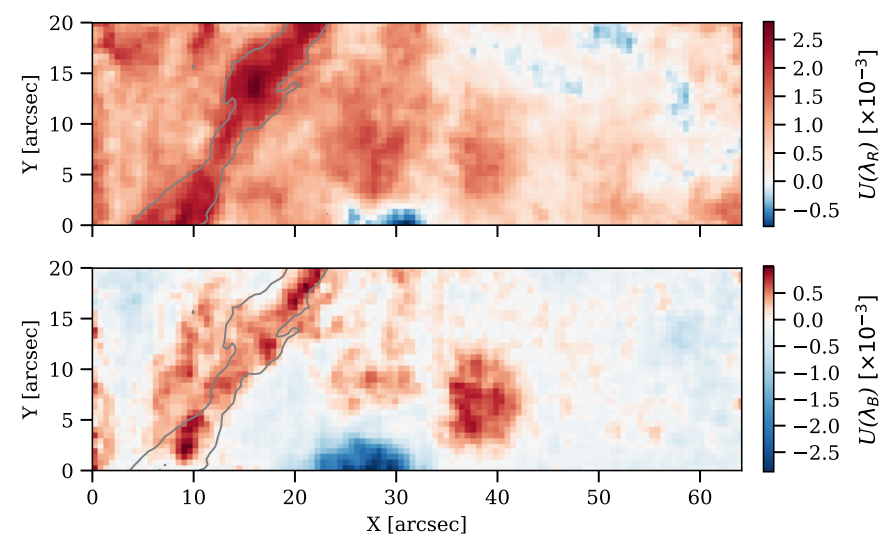

Fig. 4. Stokes $U\left(\lambda_{\mathrm{R}}\right)$ and $U\left(\lambda_{\mathrm{B}}\right)$ of the third scan. A gray contour at $0.6 I_{\mathrm{c}}$ shows the position of the filament.

$\beta$ as a free parameter to produce a better fit of Stokes $I$ leads to a slight increase in $\beta$ with a greater increase in $\tau_{\mathrm{R}}$. Figure 3 shows this clear correlation.

This experiment shows that the source function required to reproduce the profiles is greater than in the case of pure scattering $\left(S=J_{0}^{0}\right.$ and $\left.\beta=1\right)$. One possible explanation of this increase might be that the high density of the filament leads to a larger contribution of thermal processes. This idea seems feasible (also suggested by Casini et al. 2009a) because our filament has a high absorption (optical depths of up to 2 in several scans). Because HAZEL neglects collisions in the calculation of the source function (and consequently, the thermal contribution), this can lead to a reduced polarization signals. The magnetic inference in the filament can therefore be affected by this approximation.

\section{Analysis of Stokes $U$}

As mentioned in Paper I, the observations display uncommon signals in Stokes $Q$ and $U$ with the same sign in both the blue and red components. In this section, we study these signals in detail. Figure 4 displays maps of the Stokes parameter $U$ (as the polarization signals are stronger than in Stokes $Q$ ) at two different wavelengths: the central wavelength of the blue component, $\lambda_{\mathrm{B}}$ (lower panel), and the central wavelength of the red component, $\lambda_{\mathrm{R}}$ (upper panel). We focus on Stokes $U$ in the third scan because the signals are stronger. Similar results are found for the other scans.

We verified that the observed signals are not produced by any residual crosstalk from Stokes $I$ to Stokes $Q$ and $U$. Because the red component is several times stronger than the blue component in Stokes $I$, we would have detected any possible crosstalk first in the red component, and it would have been even more obvious in the silicon line.

The signals outside the filament are generated in a regime in which atomic polarization and the Zeeman effect operate simultaneously. This can be seen in the penumbra of the sunspot, where a very strong and horizontal magnetic field is present. There, two patches are easily observed in the blue component (with opposite signs), but they are more obscured in the red component. The explanation of this is that the atomic polarization affects the red component more than the blue component. Moreover, the most striking feature of Fig. 4 is that both maps present the same sign inside the filament and the opposite sign outside it. In the following, we address this problem and try to find a suitable physical scenario that can produce linear polarization 


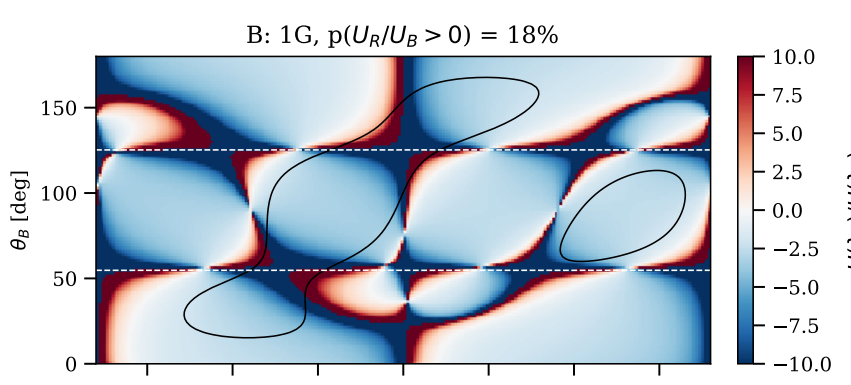

B: $100 \mathrm{G}, \mathrm{p}\left(U_{R} / U_{B}>0\right)=0 \%$

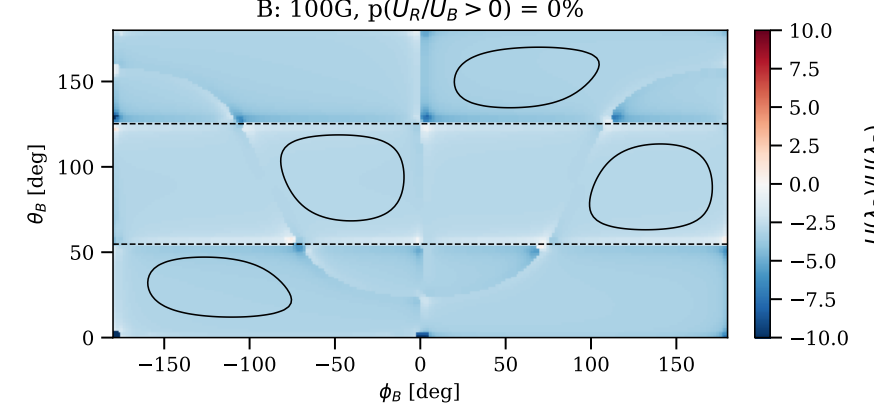

Fig. 5. Ratio between the synthetic Stokes $U$ signals at the red component $\left(\lambda_{R}\right)$ and the blue component $\left(\lambda_{B}\right)$ for a magnetic field strength of $1 \mathrm{G}$ (upper panel) and $100 \mathrm{G}$ (lower panel). Horizontal dashed lines indicate the two Van Vleck angles at $\theta_{\mathrm{B}}=54.74^{\circ}$ and $\theta_{\mathrm{B}}=125.26^{\circ}$. The label at the top of each panel indicates the area percentage that is covered by a positive ratio. Contours indicate the areas where Stokes $U\left(\lambda_{\mathrm{R}}\right)>1.5 \times 10^{-3} I_{\mathrm{c}}$.

signals with the same sign in the two components. In principle, we point out that the only way to generate both signals with the same sign with the current version of the code HAzEL is by specific magnetic field configurations. We caution that there may be other options outside the limitations of the forward modeling of HAzEL that may generate the same effect.

The lower-level Hanle effect (so called because it is produced by the populations and coherences of the lower level) can potentially create linear polarization with the same sign in the blue and red components of the He $10830 \AA$. Figure 5 shows the ratio $U\left(\lambda_{\mathrm{R}}\right) / U\left(\lambda_{\mathrm{B}}\right)$ for two different magnetic field strengths and all possible orientations of the magnetic field vector parameterized with the inclination $\theta_{\mathrm{B}}$ and the azimuth $\phi_{\mathrm{B}}$ in the local reference frame. The remaining parameters are the typical values obtained in the inversion, that is, $\tau_{\mathrm{R}}=1, \Delta v_{D}=10 \mathrm{~km} \mathrm{~s}^{-1}$, $v_{\mathrm{LOS}}=0 \mathrm{~km} \mathrm{~s}^{-1}$, and $a=0.1$. The case of $B=100 \mathrm{G}$ (lower panel of Fig. 5) shows that the ratio is relatively constant and about -3 for all configurations of the magnetic field vector. For this value of the magnetic field strength, the probability of finding the two components with the same sign is very low. In contrast, when the field is decreased to $B=1 \mathrm{G}$ (upper panel of Fig. 5), we find that $18 \%$ of the parameter space gives profiles with the same sign.

These conditions would support the idea of filaments with very weak fields in which the lower-level Hanle effect produces linear polarization of the same sign for the red and blue components. However, the amplitudes of the linear polarization signals produced for such weak fields are very small when compared with the observed typical $U\left(\lambda_{\mathrm{R}}\right)$ signals of about $1.5 \times 10^{-3} I_{\mathrm{c}}$ (marked with contours in Fig. 5). Additionally, in such cases, the synthetic Stokes $Q$ profiles have larger amplitudes than the observed signals. We therefore conclude that the linear polarization profiles cannot be explained (only) with very weak magnetic fields, with a single-component slab model and under the
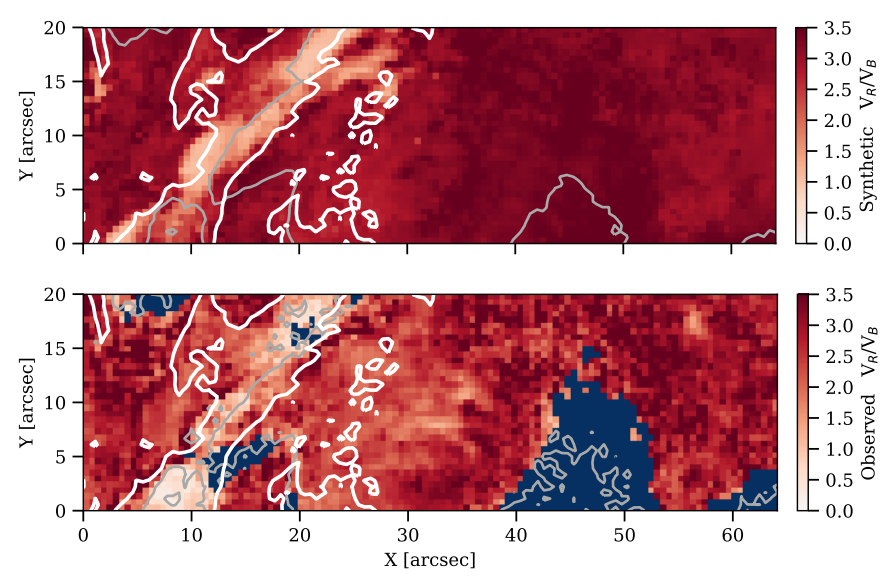

Fig. 6. Upper panel: map of the ratio of the amplitudes of the circular polarization between the red and blue components calculated using the synthetic profiles from the Hazel fit. Lower panel: same ratio, but calculated from the observed signals, masking the signals below $1.5 \sigma$. The gray line indicates the PIL, while the white contour indicates the filament location.

assumptions of the current version of HAzEL. Other plausible explanations are discussed in the conclusions.

\section{Analysis of Stokes $V$}

In Paper I we showed that the circular polarization maps in the chromosphere displayed a pattern that was very similar to the photospheric map without any indication of the presence of the filament. In addition, Díaz Baso et al. (2016) demonstrated that active region filaments, despite being dense chromospheric structures embedded in the corona, do not have enough optical thickness to completely block the light that emerges from the underlying chromosphere. At this point, it is not clear whether the magnetic field configuration of the filament resembles that of the photospheric magnetic field or if in contrast these signals came from the underlying chromosphere. In this section, we investigate the presence of more than one magnetic component in the filament region from the observed circular polarization profiles, and in particular, from the amplitude ratio of the red and blue components of the multiplet. If both components are formed under similar physical conditions, the circular polarization profiles will have a constant amplitude ratio between the two components. If this ratio has a different value, they can potentially sense different physical conditions given their different optical depths.

To this end, we computed the ratio of the Stokes $V$ amplitudes calculated in the blue lobe of the red and blue components from the observations $\left(V_{\mathrm{R}} / V_{\mathrm{B}}\right)$, which is displayed in the lower panel of Fig. 6. We chose the blue lobe because the red lobe of the red component is sometimes affected by a highly redshifted component. To compare with the single-component model inferred by Hazel, we show the same ratio for the synthetic profiles in the top panel of Fig. 6. The observed ratios shows very low values in the polarity inversion line (PIL; gray line in the figure), which are produced by noise. To avoid artifacts produced by noise, we masked all values with signals below a certain threshold. To set this threshold, we found that the estimation of the ratio is only reliable when the Stokes $V$ signals are above 1.5 times the standard deviation of the noise.

The similarities between the two maps in Fig. 6 indicate that the code is able to reproduce the observed asymmetries between the components of the line. A large part of the FOV shows 

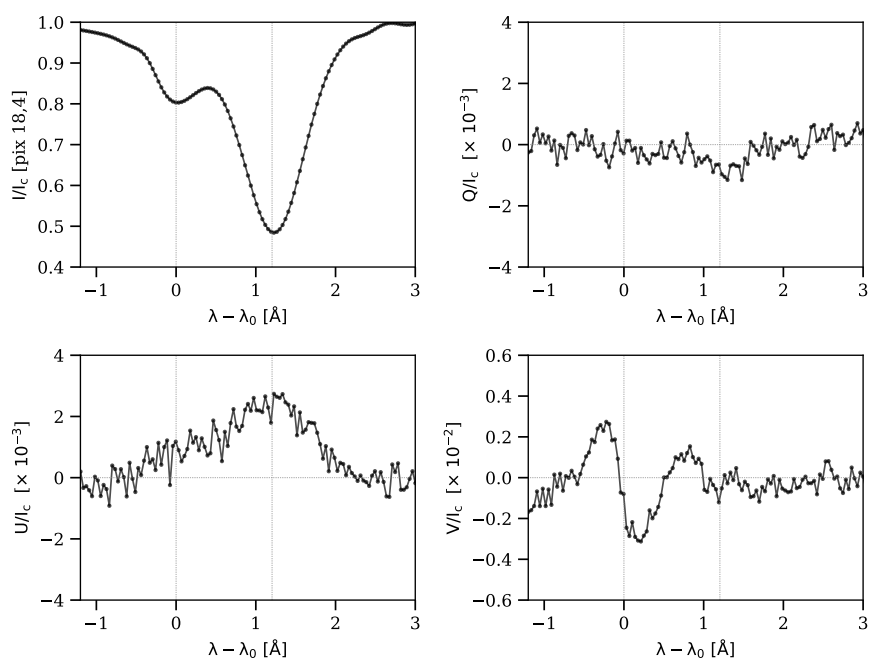

Fig. 7. Stokes profiles of the pixel $[18,4]$ with very low ratio $V_{\mathrm{R}} / V_{\mathrm{B}}$, located in the lower part of Fig. 6. The Stokes $V$ amplitude of the blue component of Stokes $V$ is twice as high as that of the red component, and $\lambda_{0}$ is the wavelength of the center of the blue component.

observed and synthetic ratios of about 3 , except inside the filament, where we find lower values (of about 1.0). The decrease of this ratio in the filament is mainly caused by the saturation of the red component, which is due to the high optical depth. A singlecomponent model seems to be sufficient to reproduce many of the filament profiles. However, we find pixels in the FOV with far lower ratios than those that can be produced by the saturation of the spectral line. The limit for a single-component atmosphere is 1 , but values as low as 0.2 are found in the observations. This indicates that more complex models with magnetic field gradients have to be used to explain these great differences. An example of this case is displayed in Fig. 7, which was extracted from the region at $\left(8^{\prime \prime}, 3^{\prime \prime}\right)$. In this figure the Stokes $V$ amplitude of the blue component is almost twice as high as that of the red component.

The question now is whether the remaining profiles, even if they do not show such clear evidence in the ratio $V_{\mathrm{R}} / V_{\mathrm{B}}$, can be explained as profiles emerging from a multicomponent model. To visualize this and to demonstrate that the saturation effect can be produced by either a single-component or a multicomponent model, we show in the following that under specific conditions, a single-component model is able to roughly reproduce the emerging profiles synthesized with a two-component atmosphere (one slab on top of the other, as in the model described in Díaz Baso et al. 2016).

For this numerical experiment, we synthesized the emergent Stokes profiles from a simulated filament (top slab) with $\tau_{\mathrm{R}}=1.2$ placed above an active region at the same heliocentric angle as the observations $(\mu=0.92)$ with a background absorption (bottom slab) of $\tau_{\mathrm{R}}=0.3$ (average value extracted from our observations). The magnetic field in the active region was parallel to the surface $\left(\theta_{\mathrm{B}}=90^{\circ}, \phi_{\mathrm{B}}=0^{\circ}\right)$ and perpendicular to the filament axis (simulating a PIL) with a strength of $B=300 \mathrm{G}$. The filament had a magnetic field strength of $B=10 \mathrm{G}$, with an azimuth of $45^{\circ}$ with respect to the field below $\left(\theta_{\mathrm{B}}=90^{\circ}, \phi_{\mathrm{B}}=-45^{\circ}\right)$. This configuration mimics the general trend of what we observe in the Stokes profiles.

The synthetic Stokes profiles are displayed in Fig. 8 together with the profiles of the fit using a single-component model. The following conclusions can be extracted from this experiment. (i) The optical depth is roughly equal to the sum of the depths
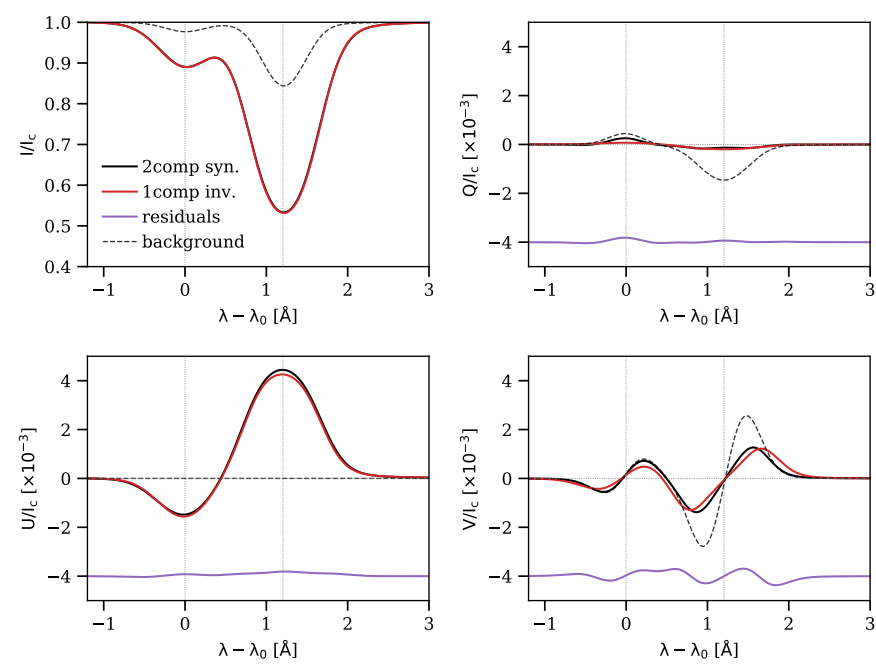

Fig. 8. Single-component model inversion fit (red) of the synthetic Stokes profiles generated with a two-component model (black). The residuals of the best fit are below each Stokes profile (purple). The dashed line indicates the Stokes signals from the background in absence of the filament.

of the two components $\tau_{\mathrm{R}} \simeq 1.5=1.2+0.3$. (ii) Although the optical depth of the filament is high, the emergent Stokes $V$ from the slab below remains almost unperturbed for the blue component after passing through the second slab, while its amplitude decreases for the red components (the original Stokes $V$ of the background is drawn as a dashed line). Some "transparency" of the plasma to circular polarization can be expected as the opacity decreases toward the wings, which is where the Stokes $V$ signals are higher, while it is almost zero in the core of the line where the absorption is very high. Because of its lower optical depth, the change is even weaker in the blue component of the He I $10830 \AA$ multiplet. This can be easily visualized assuming a Gaussian profile for the absorption with width $\sigma^{2}$. Then, the Stokes $V$ generated in the first slab will be attenuated by $V_{2}\left(\lambda_{0}\right)=V_{1}\left(\lambda_{0}\right) e^{-\tau_{2}\left(\lambda_{0}\right)}$ in the core. At the wavelengths on the Stokes $V$ lobes $\left(\lambda=\lambda_{0} \pm \sigma\right)$, the absorption is $\tau_{2}\left(\lambda_{0} \pm \sigma\right)=$ $\tau_{2}\left(\lambda_{0}\right) e^{-0.5} \sim 0.6 \tau_{2}\left(\lambda_{0}\right)$. If, for example, $\tau_{2}\left(\lambda_{0}\right)=1.2$, then $V_{2}\left(\lambda_{0} \pm \sigma\right)=V_{1}\left(\lambda_{0} \pm \sigma\right) e^{-0.6 \tau_{2}\left(\lambda_{0}\right)} \sim 0.5 V_{1}\left(\lambda_{0} \pm \sigma\right)$, which has only reduced to half. In the blue component the change is weaker because its optical depth is lower. (iii) The linear polarization detected in Stokes $U$ is mainly due to the contribution of the filament (top slab) proportional to the absorption, as we detected in the polarization maps of Paper I. The Stokes $Q$ signal generated by the background is attenuated, leaving the weak signal generated by the filament. (iv) A single-component inversion is able to reproduce the Stokes signals at a precision level of $5-10 \times 10^{-5} I_{\mathrm{c}}$, including the high asymmetry between the Stokes $V$ of the components. The residuals are well below our noise level of $4-6 \times 10^{-4} I_{\mathrm{c}}$ so that it renders these differences impossible to be detected. (iv) From the inversion we inferred a magnetic field with $B=250 \mathrm{G}, \theta_{\mathrm{B}}=78^{\circ}$, and $\phi_{\mathrm{B}}=-45^{\circ}$ for the filament, which is much stronger and more vertical than the magnetic field in the upper slab $(10 \mathrm{G})$. This has to be kept in mind in the analysis of these structures (Díaz Baso et al. 2016).

\section{Abrupt changes in the magnetic field}

In Paper I we suggested that the local azimuth map obtained from the single-component inversion displayed solutions that 

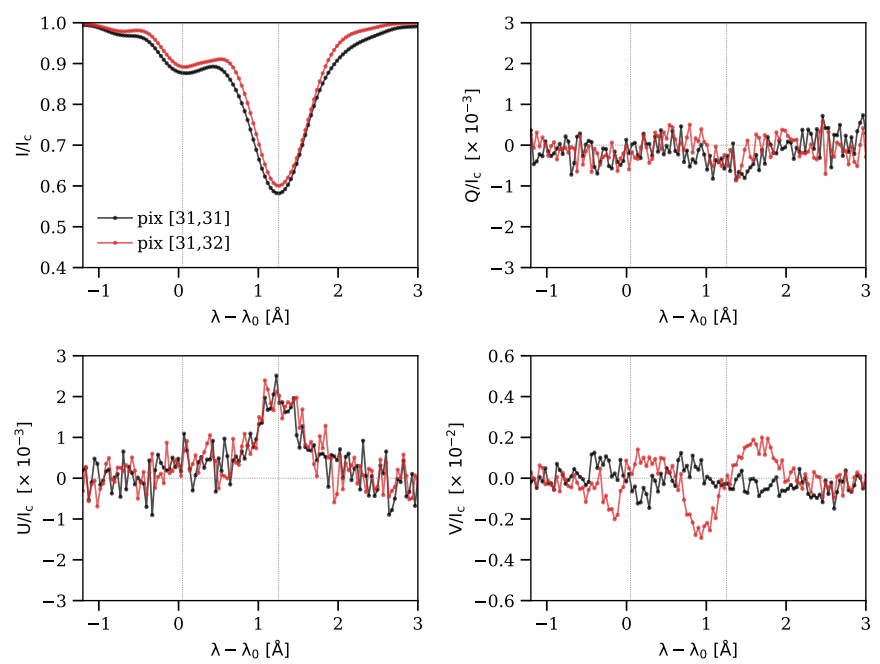

Fig. 9. Stokes profiles of the two chosen pixels from the inner part of the filament. They are two contiguous pixels at each side of the PIL. Stokes $Q$ and $U$ are very similar, while each Stokes $V$ has a different polarity.

were correlated with the polarity of the region, that is, the solutions were conditioned by the Stokes $V$ information. Moreover, in the PIL, this polarity change produced an azimuth change of up to $180^{\circ}$ within the filament. In order to understand the topology of the magnetic field correctly, we therefore need to study the distribution of the ambiguous solutions. When some of the solutions can be discarded because of their spatial distribution, the possible configuration of the filament could be obtained. In this section we analyze two contiguous pixels at each side of the PIL (i.e., from the filament axis) only taking into account Stokes $Q$ and $U$, whose signals, as we showed in the previous analysis, show the filament properties. If this abrupt change in the azimuth is real, we expect to retrieve it without the information of Stokes $V$. The Stokes profiles in these pixels are displayed in Fig. 9.

Both pixels present very similar linear polarization signals but different polarities in Stokes $V$. As in previous sections, we used MultiNest to determine the locations of the eight potential ambiguous solutions, which are displayed in Fig. 10. These eight combinations of $\theta_{\mathrm{B}}, \phi_{\mathrm{B}}$ generate the same Stokes $Q$ and $U$ profiles. Figure 10 is split into two regions of different polarities: the red region with the magnetic vector pointing toward the observer, and the blue region in the opposite direction. The red solid line shows the location of $\Theta_{\mathrm{B}}=90^{\circ}$ when it is transformed into the local reference frame $\theta=23^{\circ}$. This division also splits the eight solutions into two groups of four depending on the polarity of Stokes $V$. This implies that the local azimuth and inclination inferred would be very different only due to the polarity, even for exactly the same Stokes $Q$ and $U$ profiles.

Because drastic variations of the azimuth of the field between neighboring pixels are discouraged in the filament, smooth solutions in the full magnetic field vector might be searched for. We focused on the pair of solutions closest in local azimuth and with different polarities, the pair $[4,6]$ (Fig. 10). In this case, the two solutions differ by $\Delta \theta_{\mathrm{B}}=120^{\circ}-70^{\circ}=50^{\circ}$ in inclination. Consequently, to simultaneously fit a similar Stokes $V$ signal of opposite polarity, the magnetic field strength needs to be modified from $B=20 \mathrm{G}, \theta_{\mathrm{B}}=70^{\circ}$ to $B=200 \mathrm{G}, \theta_{\mathrm{B}}=120^{\circ}$. This increase in magnetic field strength is so large because solution 6 has a magnetic inclination in the plane of the sky close to $\Theta_{\mathrm{B}}=90^{\circ}$ (near the red line in Fig. 10). In conclusion, ensuring smoothness in the azimuth does not ensure smoothness in the inclination

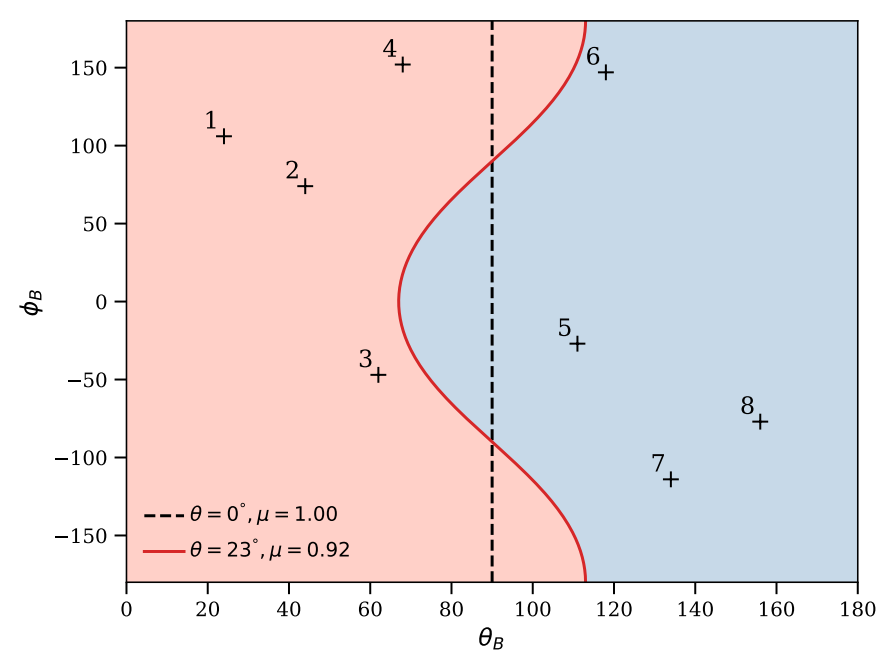

Fig. 10. Hanle ambiguities represented with pluses in the space of $\theta_{\mathrm{B}}$ and $\phi_{\mathrm{B}}$ that is compatible with both Stokes $Q$ and $U$ of the two chosen pixels of Fig. 9. The colored areas correspond to each polarity of the magnetic field. The dashed (which degenerates into a single vertical line) and continuous contours indicate the location of the polarity inversion for $\theta=0^{\circ}$ and $\theta=23^{\circ}$, respectively.

and/or the magnetic field strength. This is another indication that these Stokes profiles cannot be analyzed assuming a singlecomponent model, and we need more than one component to interpret them.

An alternative explanation for this situation is that we are detecting the two branches of a twisted flux rope at different heights that are spatially close as a result of the line-of-sight perspective. This case seems unlikely in the center of the filament (the location where these pixels are extracted) because it looks compact and homogeneous, so that the assumption a single plasma slab is plausible. The edge of the filament, where the opacity changes rapidly and where we clearly see fine structure, might be affected by this effect.

\section{Two-component inversion}

After finding in several instances that more complex models are required to explain the observed signals, in this section we explore the possibility of a two-component inversion with the idea to separate the contribution of the filament from the region below. This scenario is based on the assumption of two slabs of constant physical properties, one on top of the other, through which the emergent polarized light has to pass (as in the example of Sect. 5). In principle, although each slab can have different velocities, absorptions, and line broadenings, we reduced the dimensionality of the problem by only performing the inference over the magnetic field vector of both components: $\left(B_{1}, \theta_{1}, \phi_{1}, B_{2}, \theta_{2}, \phi_{2}\right)$. The remaining parameters were fixed to those obtained from a single-component inversion.

The two slabs had the same thermodynamic properties but different optical depths $\tau_{1}=0.3$ (value estimated from the filament surroundings) and $\tau_{2}=0.9$ (to mimic our average filament absorption). In this exercise we chose the pixel [31, 32], which was used in Fig. 9. The observed Stokes profiles and the synthetic profiles found for each mode of the $\chi^{2}$ surface are displayed in Fig. 11. It is possible to reproduce almost all observational features, such as the positive Stokes $U$ in the blue component or the complex Stokes $Q$ shape, which is impossible with a single component. 



Fig. 11. From green to blue, 10 of the 30 compatible solutions with the observed profile (gray).

However, the inference problem becomes very complex because we find very many compatible solutions, all with different configurations. In this case, we found up to 30 solutions, but only 10 are shown to avoid crowding the figure. These solutions are spread in the parameter space, and the average distance between each is about $10^{\circ}$ in the inclination and azimuth and $30 \mathrm{G}$ in the field strength $B$. The high number of solutions is a consequence of the combination of all possible ambiguities in both components and the flexibility of the two-component model in generating the same profiles with different parameter combinations.

Moreover, because of the possible cancellation of the addition of signals when the two components are crossed (Díaz Baso et al. 2016), the magnetic field can sometimes be increased (if their signals are opposite) almost arbitrarily, which means that cases may be found in which magnetic fields of $500-800 \mathrm{G}$ are required to reproduce the profiles, while a single-component inversion yields magnetic fields below $100 \mathrm{G}$. Because this analysis is multiparametric, and after extensive numerical experiments (adding or fixing other parameters), we conclude that a two-component inversion with all the free parameters is a challenging problem if there is no additional information to constrain them.

\section{Summary and conclusions}

After a first canonical analysis of an active region filament observed in the $\mathrm{He}_{\mathrm{I}} 10830 \AA$ multiplet with a single-component model, in this second part of the series we have focused our attention on observational evidence that more complex models are required to explain the observations. As the first evidence, the intensity profiles point to a very optically thick filament for which the code Hazel cannot perfectly reproduce the profiles; the code underestimates the absorption of the blue component. We fixed this using a new parameter that takes the additional emission of the slab into account. However, this improvement is not enough if radiative transfer effects cause the two components of the multiplet to be sensitive to different physical conditions.

The second evidence is the ubiquitous presence of profiles with the same sign in the blue and red components in the linear polarization inside the filament. Because a single component cannot reproduce these observational features, we referred to the proposals by Asensio Ramos et al. (2005), Trujillo Bueno \& Asensio Ramos (2007) and Judge et al. (2015) to explain it. These authors showed that when we allow for radiative transfer effects, the variation in anisotropy of the radiation field, $J_{0}^{2}$, in an optically thick slab along the line of sight can lead to the same sign in both components. The high opacity makes the red and blue components sensitive to different regions in the atmosphere with different $J_{0}^{2}$ values of opposite signs, resulting in polarization signals with the same sign. This seems to be a plausible scenario for active region filaments because they are located at low heights (where the anisotropy can change its sign more easily than at greater heights) and they have a significant optical thickness (to be sensitive to different regions in each component and promote the horizontal illumination). These two ingredients favor a negative contribution to the anisotropy because the self-emission of the slab can compete with the anisotropy of the photospheric radiation field, producing these changes of sign of $J_{0}^{2}$ with depth. Although this possibility seems realistic, the calculations of these competing effects in the previous studies still use simple assumptions. In reality, a complex multi-slab model is required that explicitly takes the variation in anisotropy along the slab and the formation of both components into account. It remains to be verified whether this effect can still hold in the complex scenario. Again, this additional evidence supports the hypothesis that the complexity of the current models needs to be increased.

The Stokes $V$ map of He I does not show any clear signature the filament, and the inferred azimuth map follows the same pattern as Stokes $V$, as if the polarity of Stokes $V$ were conditioning the inference. Using simple numerical experiments, we demonstrated here (see also Díaz Baso et al. 2016) that the filament is almost transparent to the circular polarization that is generated in the atmosphere below the filament. Its analysis as a signal coming from a single slab leads to discrepancies, such as the abrupt changes in the azimuth or assigning greater field strengths to the filament. Moreover, some indications of strong gradients of $B$ are also visible in Stokes $V$.

Finally, in an effort to study the reliability of two-component inversions with the idea of separating both contributions, we showed that the model contains too much flexibility. Although it can reproduce the observations better (better than a single component model), many compatible solutions with different configurations exist, which make the interpretation very complex. To overcome this problem and enable the analysis of the filaments, we proposed stereoscopic observations (to identify the height) and magnetic field extrapolations from the photosphere or multiline observations with the Ca II at $8542 \AA$ (Khomenko et al. 2016; Schwartz et al. 2016), for instance, to infer the magnetic field of the active chromosphere below. For these optically thick structures close or above the disk (also spicules, arches, etc.), radiative transfer modeling and additional information about the illumination from the photosphere is required as well. However, other cases where the observed line is optically thin and there is no background (such as in prominence observations using the $\mathrm{D}_{3}$ at $5876 \AA$, Casini et al. 2009b), a single component could be a plausible option. Finally, it is also important to add that noise has a strong effect on the inference process, in which a lower noise level can help reduce the number of ambiguous solutions.

The importance and misinterpretation due to oversimplified models has also been investigated by other studies in the past. An example is Milić et al. (2017), who described the bias produced when a simple $1 \mathrm{D}$ model is used to interpret a 2D inhomogeneous prominence model, retrieving more vertical and weaker magnetic field solutions. Our contribution describes the details 
that we should take into account when interpreting data from active region filaments with the next generation of telescopes. The problem is complex, and we should be prepared to deal with all problems to further improve our knowledge of the chromosphere.

Acknowledgements. The authors wish to thank Manolo Collados for useful advice on the presented work. We especially thank Andreas Lagg for observing the active region filament and providing these valuable data for us. We also wish to thank the anonymous referee for the comments and suggestions The $1.5 \mathrm{~m}$ GREGOR solar telescope was built by a German consortium unde the leadership of the Kiepenheuer Institut für Sonnenphysik in Freiburg with the Leibniz Institut für Astrophysik Potsdam, the Institut für Astrophysik Göttingen, and the Max-Planck Institut für Sonnensystemforschung in Göttingen as partners, and with contributions by the Instituto de Astrofśica de Canarias and the Astronomical Institute of the Academy of Sciences of the Czech Republic. The GRIS instrument was developed thanks to the support by the Spanish Ministry of Economy and Competitiveness through the project AYA2010-18029 (Solar Magnetism and Astrophysical Spectropolarimetry). Financial support by the Spanish Ministry of Economy and Competitiveness through projects AYA2014-60476-P and AYA2014-60833-P are gratefully acknowledged. CJDB acknowledges Fundación La Caixa for the financial support received in the form of a PhD contract. This research has made use of NASA's Astrophysics Data System Bibliographic Services. This paper made use of the IAC Supercomputing facility HTCondor (http://research.cs.wisc.edu/htcondor/), partly financed by the Ministry of Economy and Competitiveness with FEDER funds, code IACA133E-2493. We acknowledge the community effort devoted to the development of the following open-source packages that were used in this work: numpy (numpy.org), matplotlib (matplotlib.org), corner (Foreman-Mackey 2016), and SunPy (sunpy .org).

\section{References}

Antiochos, S. K., MacNeice, P. J., Spicer, D. S., \& Klimchuk, J. A. 1999, ApJ, 512,985

Asensio Ramos, A., Landi Degl'Innocenti, E., \& Trujillo Bueno, J. 2005, ApJ, 625,985

Asensio Ramos, A., Trujillo Bueno, J., \& Landi Degl'Innocenti, E. 2008, ApJ, 683,542

Bommier, V., Sahal-Brechot, S., \& Leroy, J. L. 1981, A\&A, 100, 231

Bommier, V., Landi Degl'Innocenti, E., \& Sahal-Brechot, S. 1989, A\&A, 211, 230

Casini, R., Manso Sainz, R., \& Low, B. C. 2009a, ApJ, 701, L43

Casini, R., López Ariste, A., Paletou, F., \& Léger, L. 2009b, ApJ, 703, 114
Collados, M., López, R., Páez, E., et al. 2012, Astron. Nachr., 333, 872

Díaz Baso, C. J., Martínez González, M. J., \& Asensio Ramos, A. 2016, ApJ, 822,50

Díaz Baso, C. J., Martínez González, M. J., \& Asensio Ramos, A. 2019, A\&A, 625, A128 (Paper I)

Feroz, F., Hobson, M. P., \& Bridges, M. 2009, MNRAS, 398, 1601

Foreman-Mackey, D. 2016, J. Open Source Softw., 1, 24

Joshi, J., Lagg, A., Solanki, S. K., et al. 2016, A\&A, 596, A8

Judge, P. G., Kleint, L., \& Sainz Dalda, A. 2015, ApJ, 814, 100

Keppens, R., \& Xia, C. 2014, ApJ, 789, 22

Khomenko, E., Collados, M., \& Díaz, A. J. 2016, ApJ, 823, 132

Kippenhahn, R., \& Schlüter, A. 1957, ZAp, 43, 36

Kuckein, C., Centeno, R., Martínez Pillet, V., et al. 2009, A\&A, 501, 1113

Kuckein, C., Martínez Pillet, V., \& Centeno, R. 2012, A\&A, 539, A131

Kuperus, M., \& Raadu, M. A. 1974, A\&A, 31, 189

Labrosse, N., Heinzel, P., Vial, J.-C., et al. 2010, Space Sci. Rev., 151, 243

Lagg, A., Woch, J., Krupp, N., \& Solanki, S. K. 2004, A\&A, 414, 1109

Lagg, A., Ishikawa, R., Merenda, L., et al. 2009, in The Second Hinode Science Meeting: Beyond Discovery-Toward Understanding, eds. B. Lites, M. Cheung, T. Magara, J. Mariska, \& K. Reeves, ASP Conf. Ser., 415, 327

Landi Degl'Innocenti, E. 1982, Sol. Phys., 79, 291

Libbrecht, T., de la Cruz Rodríguez, J., Danilovic, S., Leenaarts, J., \& Pazira, H. 2019, A\&A, 621, A35

López Ariste, A., \& Casini, R. 2002, ApJ, 575, 529

Mackay, D. H., Karpen, J. T., Ballester, J. L., Schmieder, B., \& Aulanier, G. 2010, Space Sci. Rev., 151, 333

Martínez González, M. J., Asensio Ramos, A., Manso Sainz, R., Beck, C., \& Belluzzi, L. 2012, ApJ, 759, 16

Martínez González, M. J., Manso Sainz, R., Asensio Ramos, A., et al. 2015, ApJ, 802, 3

Milić, I., Faurobert, M., \& Atanacković, O. 2017, A\&A, 597, A31

Orozco Suárez, D., Asensio Ramos, A., \& Trujillo Bueno, J. 2014, A\&A, 566, A46

Parenti, S. 2014, Sol. Phys., 11, 1

Penn, M. J., \& Kuhn, J. R. 1995, ApJ, 441, L51

Sasso, C., Lagg, A., \& Solanki, S. K. 2011, A\&A, 526, A42

Schad, T. A., Penn, M. J., \& Lin, H. 2013, ApJ, 768, 111

Schad, T. A., Penn, M. J., Lin, H., \& Judge, P. G. 2016, ApJ, 833, 5

Schmidt, W., von der Lühe, O., Volkmer, R., et al. 2012, in Second ATST-EAST Meeting: Magnetic Fields from the Photosphere to the Corona, eds.

T. R. Rimmele, A. Tritschler, F. Wöger, et al., ASP Conf. Ser., 463, 365

Schwartz, P., Balthasar, H., Kuckein, C., et al. 2016, Astron. Nachr., 337, 1045

Trujillo Bueno, J., \& Asensio Ramos, A. 2007, ApJ, 655, 642

Trujillo Bueno, J., Landi Degl'Innocenti, E., Collados, M., Merenda, L., \& Manso Sainz, R. 2002, Nature, 415, 403

Xia, C., Chen, P. F., \& Keppens, R. 2012, ApJ, 748, L26

Xu, Z., Lagg, A., Solanki, S., \& Liu, Y. 2012, ApJ, 749, 138 